\title{
Experimentelle Untersuchungen über Labyrintherkrankung und deren Beziehung zur Meningitis.
}

\author{
Von \\ Bronisław Karbowski. \\ (Aus dem neuro-biologischen Laboratorium [E. Flatau] der Gesellschaft \\ der Wissenschaften in Warschau.)
}

Mit 4 Textfiguren.

(Eingegangen am 25. April 1914.)

Albert Bla $\mathbf{u}^{1}$ ) hat seinerzeit über Versuche experimentellen Verschlusses des runden Fensters berichtet. Er beabsichtigte durch Plombierung der Nische zum runden Fenster den Anteil der runden Fenstermembran am Hörakt festzustellen. Die Hunde und Katzen, bei denen die Nische mit Zement plombiert wurde, zeigten dabei merkwürdige Symptome: Schiefhalten des Kopfes nach der operierten Seite, Gangstörungen und ähnliches. Dieser Symptomenkomplex legte die Vermutung an eine entzündliche Labyrinthreizung nah.

Herzog ${ }^{2}$ ) nahm aus diesem Grunde die Versuche Blaus auf, um mit Hilfe des Experimentes den Verlauf und Ablauf der Labyrinthentzündungen zu verfolgen. Durch Plombierung der Nische zum runden Fenster mit Zement, dem etwas Jod und Arsen beigemengt war, erzeugte Herzog Labyrinthentzündungen verschiedenen Grades.

In derselben Weise erhielten Labyrinthentzündungen andere Forscher.

Im Jahre 1912 versuchte Albert Bla $\mathrm{u}^{3}$ ) das Labyrinth direkt zu infizieren und führte durch die runde Fenstermembran Streptococcus erysipelatoides und Streptococcus mucosus in die Scala tympani hinein. Er erzeugte auf diese Weise seröse und eitrige Labyrinthentzündungen.

Heymann $n^{4}$ ) in seiner Arbeit: „Experimentelle Untersuchungen über die akuten Mittelohrentzündungen" hat eine ganze Reihe von

1) Blau, Albert, Experimenteller Verschluß des runden Fensters. Verh. d. otol. Ges. 1905 .

2) Herzog, Experimentelle Labyrinthitis. Sitzber. d. Ges. f. Morph. u. Phys. Münch. 1908.

3) Bla u, Experimentelle Studien über die Labyrinthitis. Archiv f. Ohrenheilk. 40. 1912.

4) Heymann, Experimentelle Untersuchungen über die akute Mittelohrentzündung. Archiv f. Ohrenheilk. 1913. 
Fällen veröffentlicht, in welchen experimentell erzeugte Mittelohrentzündungen auf das Labyrinth hinübergegangen sind.

In der vorliegenden Arbeit kam es mir darauf an, die Labyrinthentzündungen meningogener Entstehung in Experimenten kennenzulernen.

Als Material zu dieser Arbeit dienten mir Hunde, an denen Flata $u$ und Handelsmann experimentelle Genickstarre erzeugten.

Als Erreger sind verschiedene Pneumokokkenstämme verwendet worden.

Görke ${ }^{1}$ ) in seiner Arbeit: „Die entzündlichen Erkrankungen des Labyrinthes" wies darauf hin, daß das Studium von Labyrinthentzündungen meningogener Art für die Otopathologie von großem Wert sein kann. Er hob hervor, daß bei der meningogenen Labyrinthitis das Alter der im Labyrinthe konstatierten Veränderungen mit größerer Genauigkeit zu bestimmen sei, als es bei den von der Paukenhöhle her induzierten Labyrintherkrankungen möglich wäre; daß die meningogenen Formen vieles liefern können, was zur Deutung der Bilder tympanaler Entstehung verwertet werden kann; endlich, daß für die Frage der Fortleitung der Entzündungen vom Labyrinthe auf die Meningen, das Studium der meningitischen Formen von Wert sein kann, da dieselben Wege, wenn auch in umgekehrter Richtung, in Betracht kommen.

Aus diesen Gründen scheint mir die Mitteilung über die Ergebnisse meiner histologischen Untersuchungen berechtigt zu sein.

Meine Untersuchungen beziehen sich auf 12 Fälle. Von diesen waren in drei Fällen keine pathologischen Veränderungen vorhanden; es fanden sich auch keine Veränderungen an den Hirnhäuten. In den übrigen 9 Fällen, in welchen klinisch und anatomo-pathologisch Hirnhäuteentzündungen festgestellt worden sind, fand ich seröse und seröseitrige Veränderungen im inneren Ohre. In 8 Fällen waren die Veränderungen doppelseitig; in einem Falle nur im rechten Labyrinth.

Wir sehen also, da B in sämtlichen Fällen, wo das Bild der Meningitis zur vollen Entwicklung kam, der entzündliche Prozeß von den Hirnhäuten auf das Labyrinth hinübergegangen ist. Bezüglich des Weges hat es sich herausgestellt, daß der Uberleitungsweg ausschließlich der Aquaeductus cochleae war (Fig. 1). In sämtlichen Fällen wurde im Aquaeductus cochleae Eiter gefunden; in seinem endostalen Teile — Quellung und Hyperämie; der Bindegewebsstrang, der den größten Teil des Lumens ausfüllt, war stark mit Eiterzellen infiltriert. In der Schnecke waren an der Einmündungsstelle des Aquaeductus cochleae die entzündlichen Erscheinungen am stärksten ausgesprochen. In sämtlichen Fällen war in der Basalwindung zellreiches

1) Görke, Archiv f. Ohrenheilk. 80, H. 1-2. 1909. 
eitriges Exsudat zu sehen; in der mittleren und oberen Windung nahm die Intensität der Entzündung bedeutend ab. Für den Utbergang der Entzündung durch die Schneckenwasserleitung spricht außerdem auch die starke Mitbeteiligung der runden Fenstermembran. Schon in den Fällen, wo es sich um den ersten Moment des Einbruches handelte, sind die Veränderungen an der Membran stark ausgesprochen.

Daß der Aquaeductus cochleae der ausschließliche Weg war beim Ubergang der Entzündung von den Meningen auf das Labyrinth, dafür

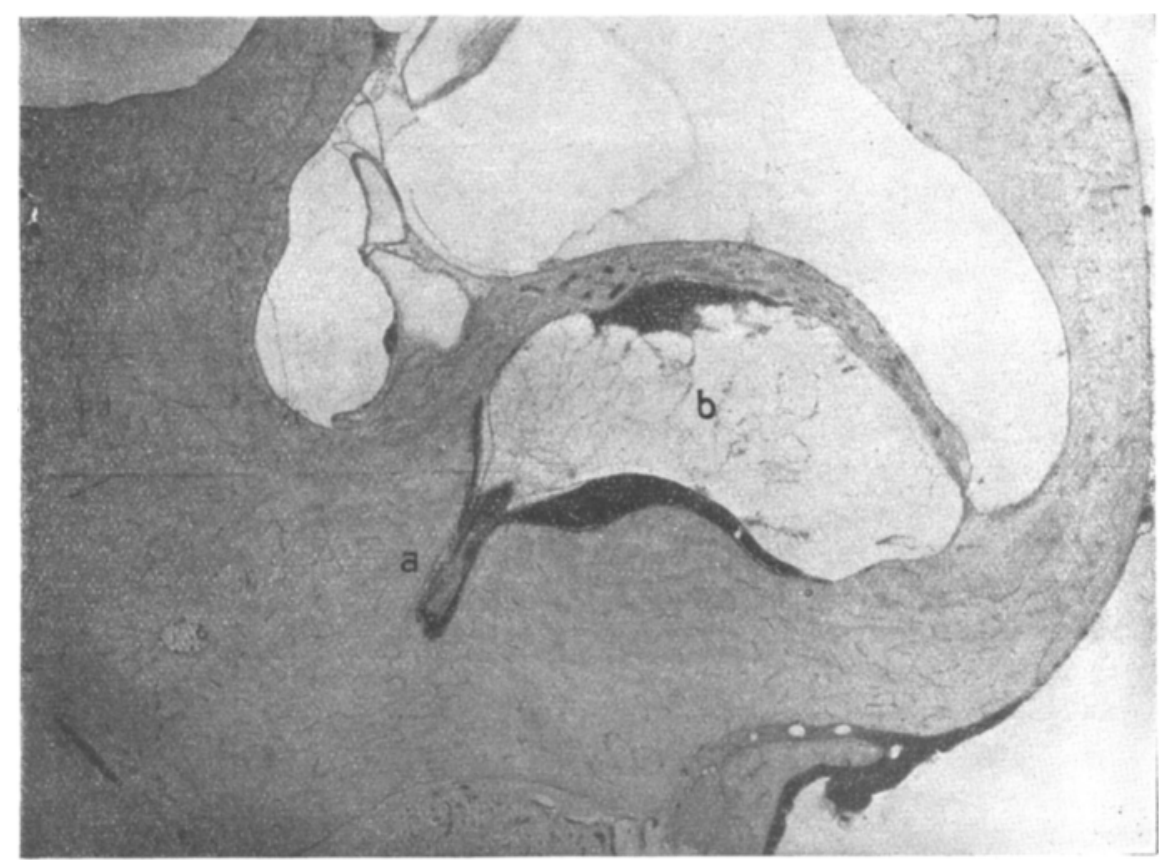

Fig. 1. $a$ = eitrige Entzündung des Aquaeductus cochleae; $b=$ Scala tympani.

sprechen auch die Befunde am Meatus acusticus internus. Man sah hier und da leichte Infiltration der Nervenscheiden; nirgends überschritt aber die Infiltration die Grenzen des Fundus.

Im Modiolus der Schnecke fand man in den weit fortgeschrittenen Fällen eine bedeutende Hyperämie der Nerven, nirgends aber nennenswerte eitrige Infiltration. In mehreren Fällen wurde der Aquaeductus vestibuli in den gefärbten Schnitten gefunden; es waren aber keine entzündlichen Veränderungen feststellbar.

Wenn wir die statistischen Angaben über die Mitbeteiligung des inneren Ohres in Meningitisfällen beim Menschen mit den von mir 
erhobenen Befunden bei Hunden vergleichen, so fällt eine starke Differenz auf.

Beim Menschen wurde die Mitbeteiligung des Labyrinthes weniger als in der Hälfte der Fälle festgestellt; in Experimenten an Hunden in sämtlichen Fällen. Verschieden sind auch die Wege, auf denen die Entzündung beim Menschen und experimentell erzeugten Genickstarrefällen auf das innere Ohr hinübergeht. Beim Menschen wird der Meatus acusticus internus bevorzugt. Der entzündliche Prozeß schreitet durch die perivasculären und perineuralen Lymphräume; in seltenen Fällen durch den Aquaeductus cochleae. Bei unseren Hunden war der Aquaeductus coohleae der einzige Utberleitungsweg.

Die Ursache dieses Unterschiedes ist in den anatomischen Verhältnissen zu suchen. Der Aquaeductus cochleae ist beim Menschen bedeutend länger und schmäler als beim Hunde. Die Länge der Wasserleitung beträgt beim Menschen $13 \mathrm{~mm}$, bei Hunden, nach cigenen Messungen, 4-5 mm. Außerdem ist der Aquaeductus cochleae beim Hunde mit einem lockeren bindegewebigen Strange versehen, der, nach eigenen Untersuchungen an normalen Hunden, von der Arachnoidea seinen Ursprung nimmt.

Es folgt daraus, daß nicht nur die Dimensionen des Aquaeductus, sondern auch der bindegewebige Strang den Utbergang von den Meningen auf das Labyrinth erleichtern.

Ebenfalls in den anatomischen Verhältnissen ist die Ursache zu suchen, warum in keinem einzigen Falle der entzündliche Prozẹ von den Meningen auf das Labyrinth durch die perivasculären und perineuralen Lymphräume hinübergegangen ist. Während beim Menschen die perivasculären und perineuralen Lymphräume weit und geräumig sind und oft ganz nah den Skalen anliegen, sind beim Hunde die Nervenkanäle durch den stark entwickelten $\mathrm{N}$. cochlearis und vestibularis fast vollständig ausgefüllt, so daß die perineuralen Lymphräume im Modiolus oft vollständig fehlen; die perivasculären Lymphräume sind ebenfalls unbedeutend entwickelt. Beim Hunde finden wir auch nirgends zarte bindegewebige Membranen zwischen den perilymphatischen Räumen und den Skalen, sondern ziemlich dicke Knochenlamellen.

Was die pathologisch-anatomisohen Bilder betrifft, so sind sie im großen und ganzen so ähnlich, daß einer allgemeinen Beschreibung nichts im Wege steht.

In sämtlichen Fällen, ausgenommen diejenigen, in welchen der Prozeß länger als 48 Stunden dauerte, fand man in der basalen Windung der Scala tympani reichliches, eitriges Exsudat. Der endostale Auszug an derselben Windung stark hyperämisch, stellenweise infiltriert. Die Venen des Aquaeductus cochleae stark erweitert; in manchen Fällen in der Umgebung - Hämorrhagien (Fig. 2). In der mittleren Windung 
derselben Skala ist das Exsudat noch ausgesprochen eitrig, der Zellreichtum nimmt aber bedeutend $a b$; in der oberen Windung ist der Inhalt serös. In der Scala vestibuli finden wir ausschließlich seröse Ausschwitzungen; hier und da kleine Gruppen von Eiterkörperchen. Im Ductus cochlearis sind bedeutende Veränderungen im oberen Abschnitte zu sehen; in der mittleren und basalen Windung finden wir nur ge-.

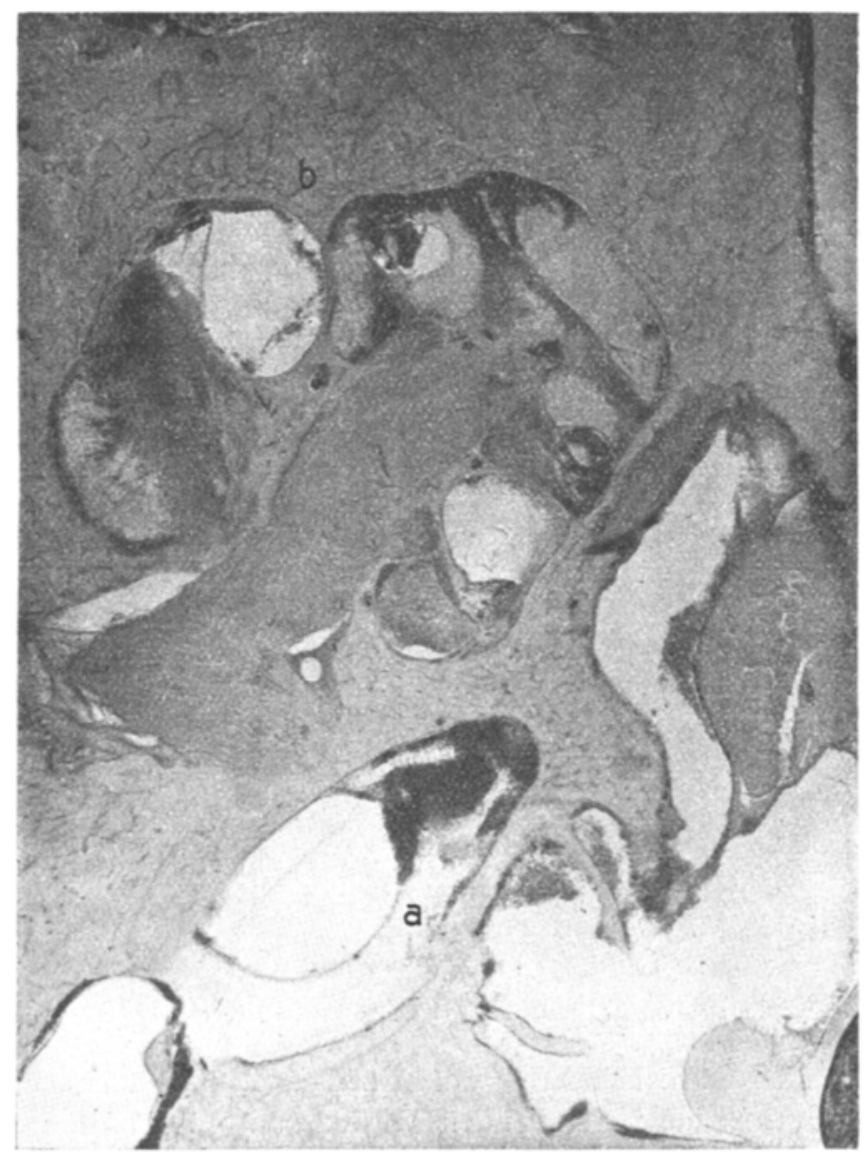

Fig. 2. Diffus $\theta$ eitrige Entztindung des Labyrinthes ( $a=$ Vestibulum; $b=$ Schnecke).

ringe entzündliche Veränderungen. Hier ist die Stria vascularis vollständig normal, die Cortische Membran in ihrer Konfiguration nicht verändert, das Cortische Organ nur wenig geschädigt.

Ein ganz anderes Bild stellt der obere Abschnitt dar. Das Ligamentum spirale stark hyperämisch, die Stria vascularis zerstört durch Hämorrhagien; im Lumen des Ductus - serös-hämorrhagisches Ex- 
sudat. Die Membrana tectoria ist in einzelnen Fällen so stark gequollen, daß sie das Lumen ganz ausfüllt. Das Cortische Organ ist stark geschädigt. Die Reissnersche Membran ist vorgewölbt.

Im Vestibulum waren die entzündlichen Veränderungen hauptsächlich perilymphatisch stark ausgesprochen (Fig. 3). Im unteren Abschnitt, speziell um den Utriculus, fand sich serös-eitriges Exsudat mit reichlicher Blutbeimengung. Das perilymphatische Bindegewebe ist stark hyperämisch und mit Rundzellen infiltriert. Endolymphatisch im Sacculus und Utriculus feinkörnige Niederschläge; an den

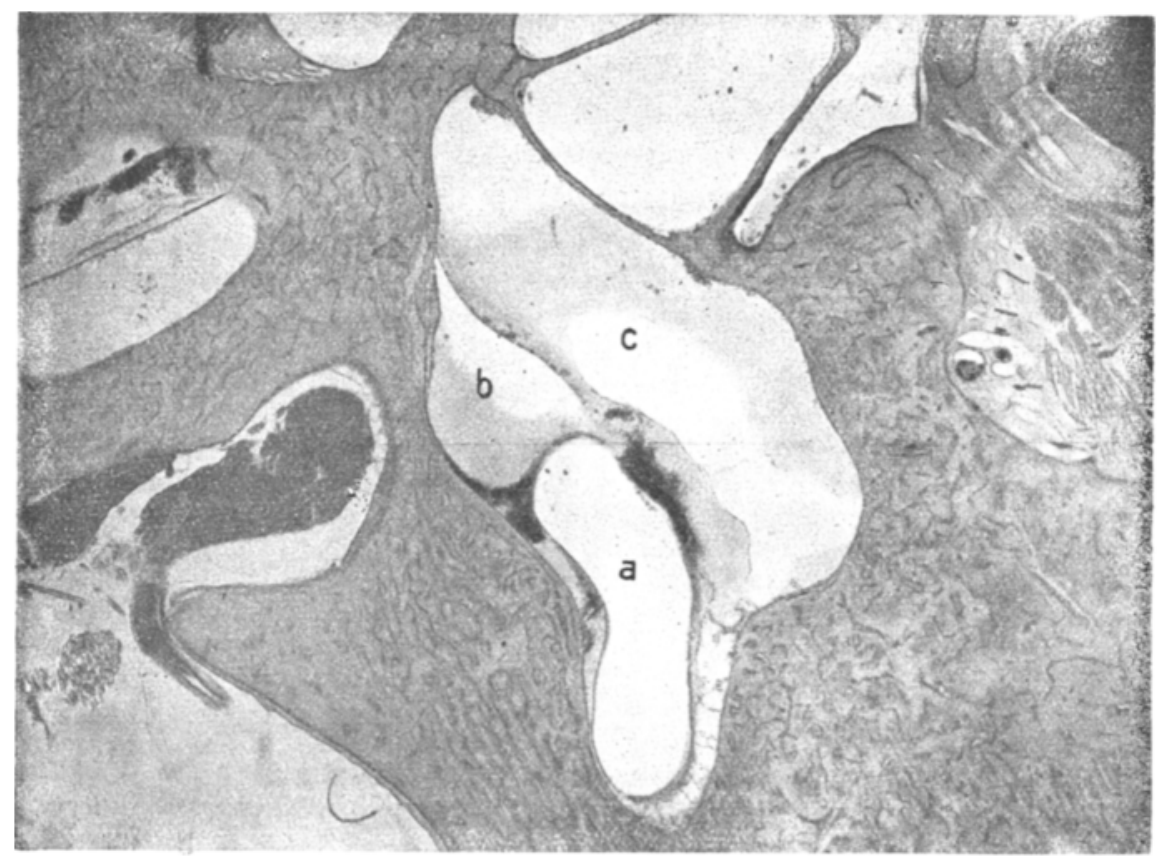

Fig. 3. Serös-eitrige Entzündung im Vestibulum ( $a$ =Utriculus; $b=$ Sacculus; $c=$ perilymphat. Raum).

Wandungen seröse Sichel, selten waren zellige Elemente zu sehen. Die Maculae zeigen keine tiefgreifenden Veränderungen.

In den Bogengängen waren die entzündlichen Veränderungen gering; nur perilymphatisch - seröser Inhalt. Weit fortgeschrittene Veränderungen zeigte die Ampulle des hinteren Bogenganges; bedeutende Zellinfiltration und Hämorrhagien.

Was die Fenster anbelangt, so war das ovale Fenster frei von entzündlichen Veränderungen: das Ligamentum ovale zeigte nirgends etwas Pathologisches; dagegen war die runde Fenstermembran stark entzündlich verändert. Sämtliche Schichten waren dicht mit Eiter- 
zellen infiltriert; sie bildeten auch den ťberleitungsweg, durch den die Entzündung vom inneren auf das mittlere $\mathrm{Ohr}$ hinübergegangen ist.

Im Mittelohr finden wir in allen Fällen, die nicht länger als 38 Stunden im Versuch standen, meist eine eitrige Entzündung, beschränkt auf den Abschnitt, der der runden Fenstermembran anliegt.

Ein ganz anderes Bild finden wir in den Fällen, die mehrere Tage im Versuche standen. Es sind in diesen Fällen weit fortgeschrittene, diffuse eitrige Entzündungen vorhanden mit starken Zerstörungen, speziell des häutigen Labyrinthes.

$\mathrm{Zu}$ erwähnen sind noch die Fälle, die mit Urotropin behandelt worden sind. Auffallend ist an diesen Fällen der stark ausgesprochene hämorrhagische Charakter der Entzündung. Besonders reichlich waren die Blutungen im basalen Abschnitte der Schnecke und in der oberen Windung. Im Vestibulum waren stärkere Blutansammlungen - in der Umgebung des Utriculus und an der Ampulle des hinteren Bogenganges. In diesen Fällen waren auch in den Hirnhäuten starke Blutextravasate vorhanden.

Aus denjenigen Fällen, in denen der Entzündungsprozeß nicht weit fortgeschritten war, können wir schließen, wie die Entzündung im Labyrinthe sich verbreitet. Dies gilt selbstverständlich nur für die Fälle mit ausgesprochenem, akuten Verlauf und in denen der Einbruch in die basale Windung stattfand.

In der Cochlea verbreitet sich der Prozeß per continuitatem. Der Entzündungsprozeß schreitet von der basalen Windung der Scala tympani auf die mittlere Windung, von der mittleren auf die obere und dann durch das Helicotrema auf die Scala vestibuli. Der Ductus cochlearis leistet anfangs dem infektiösen Prozeß einen beträchtlichen Widerstand. Stärkere Veränderungen findet man bei den Frühformen nur im Ductusabschnitt der oberen Windung; es ist aber der Ausdruck einer venösen Stauung, worüber weiter unten die Rede sein wird. Auffallend sind die ziemlich starken Veränderungen im Vestibulum, die per continuitatem von der Einbruchsstelle nicht entstehen könnten. Es mußte also der Blutweg gewesen sein, auf dem der Prozeß sich verbreitet hat. Eine Rolle spielt hier sicher die Art. vestibulocochlearis, die 1/3 der unteren Schneckenwindung, die Spitze der Schnecke, die unteren Abschnitte des Sacculus und Utriculus, endlich die Ampulle des hinteren Bogenganges versorgt. Der Art. vestibulocochlearis entsprechende Vene ist die Vena aquaeducti cochleae.

Die Verzweigungen der Venen erklären uns die Stauungserscheinungen an den schon früher erwähnten Stellen: an der oberen Windung, in der Basalwindung, am unteren Abschnitt des Sacculus und Utriculus, auch an der Ampulle des hinteren Bogenganges.

Die Strömungsverhältnisse in der Vena aquaeducti cochleae waren 
164 B. Karbowski: Experimentelle Untersuchungen über Labyrintherkrankung

durch den entzündlichen Prozeß der Umgebung stark beeinträchtigt. Der Abfluß war, wenn nicht auf ein Minimum reduziert, sicher bedeutend erschwert. Der mangelhafte Abfluß also erklärt uns die Stauungserscheinungen an allen diesen Stellen, von denen aus das Blut durch die Vena aquaeducti cochleae abfließt.

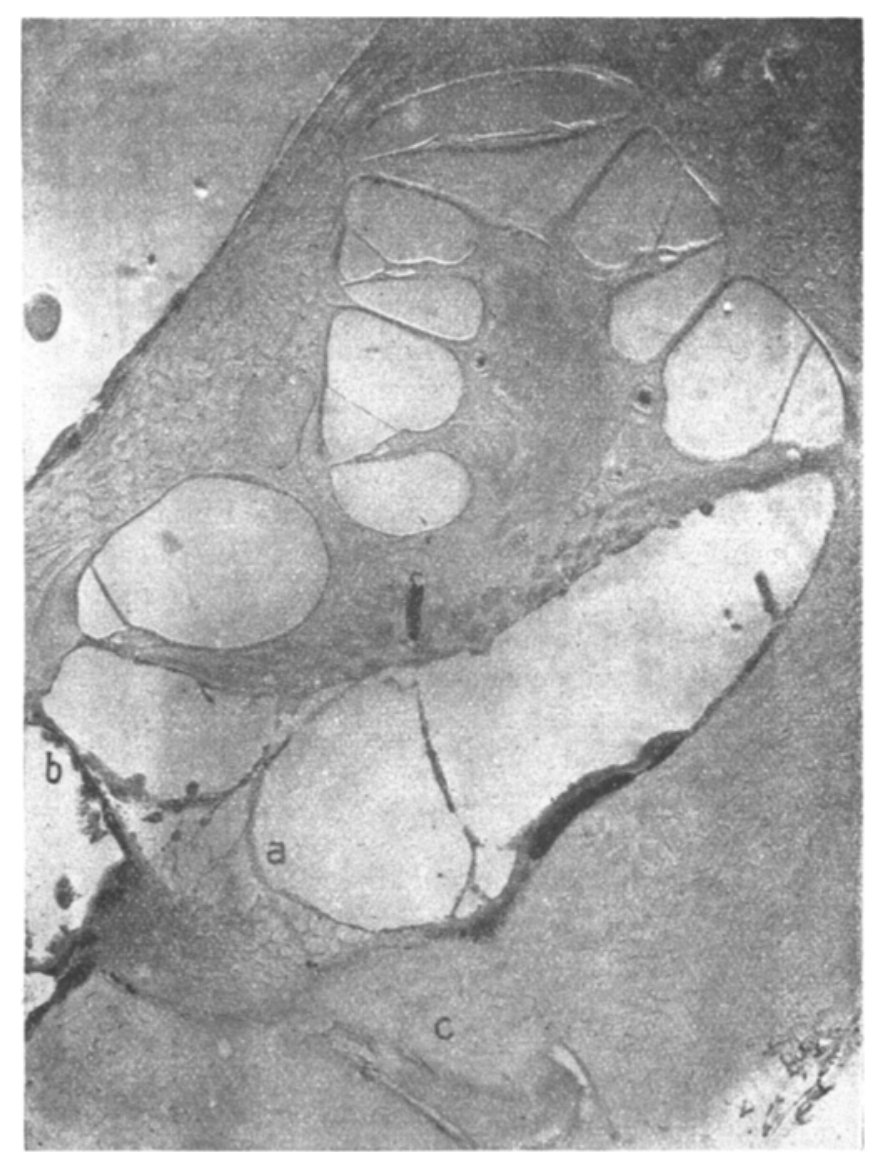

Fig. 4. Circumscripte eitrige Entzündung im basalen Abschnitte der Schnecke ( $a=$ Entzíndungsherd; $b=$ runde Fenstermembran; $c=$ Aquaed. cochl.).

Die Untersuchungen liefern auch einen Beitrag zu der Frage der primären circumscripten Labyrintheiterungen.

In einem Falle war der Prozeß nur auf die Schnecke beschränkt, das Vestibulum war vollständig frei. Im zweiten Falle war ein Entzündungsherd im basalen Abschnitte der Scala tympani und an der runden Fenstermembran. 
Namentlich der letzte Fall ist beachtenswert, da der übrige Teil der Schnecke und das Vestibulum vollständig frei waren nicht nur von pathologischen Veränderungen, sondern auch von postmortalen (Fig. 4).

Die von mir untersuchten Fälle liefern auch einen Beitrag zur Frage der Ektasie des häutigen Labyrinthes bei entzündlichen Prozessen.

Ektasien des häutigen Labyrinthes sind in Fällen beobachtet worden, wo es sich um langsam durch die Labyrinthwand fortschreitende Entzündungen gehandelt hat. Bei akut entstandenen Labyrinthitiden sollen Ektasien nicht vorkommen. In tbereinstimmung mit Albert Bla u stellte ich in einigen meiner Fälle Ektasien speziell des Ductus cochlearis fest.

Die Erweiterung des häutigen Labyrinthes in meinen Fällen ist sehr auffallend, da der Aquaeductus cochleae stark entzündlich verändert war und teilweise obliteriert gewesen war. Die Unwegsamkeit des Aquaeductus sollte eher zur Kollabierung des häutigen Labyrinthes führen, da der Abfluß der Perilymphe nicht mehr stattfinden konnte. Es ist daraus der Schluß zu ziehen, daß beim Zustandekommen von Ektasien der Schneckenwasserleitung keine Rolle zukommt.

Meine Untersuchungen erlauben folgende Schlüsse.

1. Bei experimentell hervorgerufener Cerebrospinalmeningitis an Hunden geht der entzündliche Prozeß in sämtlichen Fällen auf das Labyrinth über.

2. Der Prozeß geht ausschließlich durch den Aquaeductus cochleae auf das Labyrinth über.

3. Im Labyrinth verbreitet sich der Prozeß in der Schnecke per continuitatem; der UUbergang auf den Vorhof findet auf dem Blutwege statt.

4. Primär circumscripte Labyrintheiterungen kommen vor.

5. Ektasien des häutigen Labyrinthes bei akuten Prozessen sind möglich.

6. Die Unwegsamkeit des Aquaeductus cochleae ruft keine Kollabierung des häutigen Labyrinthes hervor. 